\title{
DETERMINACIÓN DE CINC A MICROESCALA EN UN MEDICAMENTO ESPECIFICO
}

\author{
Oscar A. Díaz* y Elida Alvarez
}

Facultad de Ingeniería, Universidad Nacional del Centro de la Provincia de Buenos Aires, Avda. Del Valle 5737, B7400JWI Olavarría, Argentina

Recebido em 7/5/03; aceito em 13/4/04; publicado na web em 19/7/04

\begin{abstract}
MICRO-SCALE ZINC DETERMINATION IN A SPECIFIC MEDICINE. This work presents zinc determination in certain medicines that contain zinc oxide and zinc undecylenate. The technique consists of a spectrophotometric micro-scale titration, where EDTA is used as titrant, and xylenol orange as an indicator, in a medium adjusted to $\mathrm{pH}=6$ with acetic acid and sodium acetate. After each added portion of EDTA, the absorbance value is measured at a selected wavelength, in order to detect the end-point of the spectrophotometric titration. The results already obtained are satisfactory and promote student's interest. An additional contribution intends to propose the use of micro-scale techniques.
\end{abstract}

Keywords: spectrophotometric titration; EDTA; xylenol orange.

\section{INTRODUCCCIÓN}

El cinc metálico no se encuentra de forma natural en el medio ambiente. Está presente sólo en estado bivalente como Zn (II). El cinc iónico sufre solvatación, dependiendo su solubilidad del pH y de la concentración de aniones. El cinc es un elemento de transición y puede formar complejos con diversos ligandos orgánicos. En la naturaleza no existen compuestos organometálicos de cinc.

Los compuestos de cinc inorgánico tienen varias aplicaciones, por ejemplo de equipamiento para el automóvil, baterías de acumuladores, pilas secas, y aplicaciones dentales, médicas y domésticas. Existen compuestos orgánicos de cinc que se utilizan como fungicidas, antibióticos tópicos y lubrificantes ${ }^{1}$.

En los sistemas biológicos el cinc está claramente perfilado como un elemento esencial para el normal desarrollo y crecimiento así como para los procesos de reconstitución de tejidos y aparece también involucrado en la química y fisiología del cerebro y el sistema nervioso ${ }^{2}$. El óxido de cinc ejerce una actividad astringente leve sobre la piel y forma una capa protectora contra la excoriación y otros agentes agresivos externos, el undecilenato de zinc es un agente cicatrizante y germicida.

Para la determinación del cinc las técnicas instrumentales normalmente utilizadas son: métodos espectrofotométricos ${ }^{3,4}$, espectrometría de emisión atómica de plasma con acoplamiento inductivo ${ }^{5}$, espectrometría de absorción atómica ${ }^{3}$, voltametría de arranque anódico y catodico ${ }^{6}$.

Las técnicas a microescala permiten optimizar recursos, reducir la generación de residuos, concientizar y promover la cultura ecológica y mejorar la seguridad en el ámbito del laboratorio. Por las mencionadas características son de interés permanante ${ }^{7-9}$.

La estrategia de trabajar a microescala consiste en reducir los niveles de experimentación a valores de 25 a $150 \mathrm{mg}$ para sólidos y de 100 a $2000 \mu \mathrm{L}$ para líquidos ${ }^{10}$.

El objetivo de este trabajo es lograr una participación activa de los estudiantes en temas que ya han sido tratados con anterioridad, sumado a una experiencia de laboratorio que permite enriquecer los conocimientos adquiridos y desarrollar sus habilidades desde el punto de vista experimental.

*e-mail: odiaz@fio.unicen.edu.ar
La experiencia de laboratorio propuesta es un método alternativo para la determinación de $\mathrm{Zn}$ en un medicamento específico, para ser utilizado en un curso de Química Analítica. Este es un experimento que permite a los estudiantes poner en práctica conceptos ya aprendidos como: complejos; espectrofotometría; titulaciones espectrofotométricas.

El método consiste en una microtitulación espectrofométrica, utilizando como agente titulante EDTA y naranja de xilenol como indicador. El $\mathrm{Zn}$ puede ser detectado en pequeñas cantidades, valorando en forma directa con EDTA y el indicador mencionado ${ }^{11}$.

Han sido descriptos numerosos métodos para la determinación de $\mathrm{Zn}$ en muestras diversas, usando agentes complejantes ${ }^{12-15}$. La reacción del $\mathrm{Zn}$ con el naranja de xilenol forma un compuesto 1:1 de color amarillo-verdoso a un pH óptimo 5,8 - 6,2 con un máximo de absorción a $570 \mathrm{~nm}$. La constante de estabilidad del complejo fue establecida a $\mathrm{pH}=6$ en medio regulado de ácido acético - acetato de sodio $^{12}$ en un valor de $\mathrm{K}_{\mathrm{Zn}-\mathrm{XO}}=1,40 \times 10^{6}$. Por otra parte la reacción del $\mathrm{Zn}$ con el EDTA forma también un compuesto 1:1 incoloro y el valor de su constante de estabilidad ${ }^{16}$ es de $\mathrm{K}_{\text {Zn-EDTA }}=3,16 \times 10^{16}$. Las interferencias que se presentan se deben a $\mathrm{Fe}(\mathrm{III}), \mathrm{Al}(\mathrm{III}), \mathrm{Pb}(\mathrm{II})$, $\mathrm{Cu}$ (II) pudiendo ser enmascaradas los dos primeros, con fluoruro de sodio y los restantes eliminados por electrólisis ${ }^{16}$.

La absorción de luz, es una de las diversas propiedades físicas cuyo cambio se puede utilizar para seguir el progreso de una titulación. Para aplicar un método de titulación espectrofotométrico es necesario que uno o más de los reactivos o de los productos absorban la radiación, o que la titulación se haga en presencia de un indicador absorbente. En las titulaciones espectrofotométricas se registran la transmitancia o absorbancia de la solución en una longitud de onda adecuada mientras se va añadiendo el reactivo titulante.

\section{PARTE EXPERIMENTAL}

\section{Equipamiento y accesorios}

Espectrofotómetro UV- Visible. Metrolab 1700. Balanza analítica, Mettler AE 240. Micropipeta $100 \mu \mathrm{L}$ Eppendorf, serie 2100. Cubetas de $1 \mathrm{~cm}$ de paso de luz de vidrio ó plástico. Vasos de precipitados. Matraces aforados por 100 y $1000 \mathrm{~mL}$. Pipetas graduadas de 2 y $5 \mathrm{~mL}$. Tubos de ensayos. Buretas por $50 \mathrm{~mL}$. Parafilm M American National Can. 


\section{Reactivos y soluciones}

Todos los reactivos utilizados fueron de grado analítico, marca Merck. Solución de EDTA 0,005370 $\mathrm{mol} \mathrm{L}^{-1}$ estandarizada con 50,00 $\mathrm{mL}$ de una solución patrón de $\mathrm{Zn}^{2+} 0,002460 \mathrm{~mol} \mathrm{~L}^{-1}$ y $0,25 \mathrm{~mL}$ de naranja de xilenol al $0,5 \%(\mathrm{~m} / \mathrm{v})$ como indicador y $2,00 \mathrm{~mL}$ de solución reguladora $\mathrm{pH}=6$ de ácido acético - acetato de sodio ${ }^{17}$. Solución patrón de $\mathrm{Zn}^{2+} 0,002460 \mathrm{~mol} \mathrm{~L}^{-1}$ preparada disolviendo $200 \mathrm{mg}$ de $\mathrm{ZnO}$ con 1,50 mL HCl $6 \mathrm{~mol} \mathrm{~L}^{-1}$ y completando el volumen a $1000 \mathrm{~mL}$ con agua destilada en matraz aforado. Naranja de xilenol al $0,5 \%(\mathrm{~m} / \mathrm{v})$, solución acuosa. Solución reguladora $\mathrm{pH}=6$ de ácido acético - acetato de sodio $^{16}$, preparada mezclando $47.30 \mathrm{~mL}$ de solución de $\mathrm{NaCOOCH}_{3}$ 0,2000 mol L-1 y 2,65 mL de $\mathrm{CH}_{3} \mathrm{COOH}$ $0,2000 \mathrm{~mol} \mathrm{~L}^{-1}$ para obtener un volumen final de $50,00 \mathrm{~mL}$. Polvo comercial cicatrizante Farm X.

\section{Procedimiento}

Para realizar la microtitulación espectrofotométrica, en primer lugar se obtienen los espectros de absorción de una solución agua destilada - naranja de xilenol y de una solución de cinc - naranja de xilenol, con el fin de determinar la longitud de onda de máxima absorción para ambas soluciones, de esta manera se establece la longitud de onda de trabajo y el cambio de color del indicador para observar el punto final de la titulación.

Para ello en un tubo de ensayo, se toman $2,00 \mathrm{~mL}$ de agua destilada, $0,50 \mathrm{~mL}$ de solución reguladora $\mathrm{pH}=6$ y $0,10 \mathrm{~mL}$ de naranja de xilenol al $0,5 \%(\mathrm{~m} / \mathrm{v})$. Se procede de igual manera para 2,00 $\mathrm{mL}$ de solución de cinc $0,002460 \mathrm{~mol} \mathrm{~L}^{-1}$. Las soluciones así obtenidas se colocan en cubetas de $1 \mathrm{~cm}$ de paso de luz del espectrofotómetro Metrolab 1700 y se obtiene el espectro de absorción de ambas soluciones, entre 380 y $660 \mathrm{~nm}$, utilizando como blanco agua destilada.

En la Figura 1 se observan ambos espectros superpuestos en la región mencionada. Esta información permite establecer la longitud de onda de trabajo en $414 \mathrm{~nm}$.

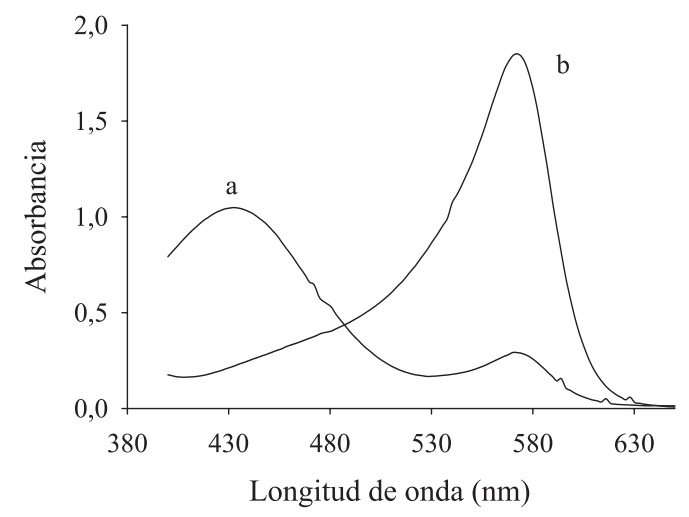

Figura 1. Espectro de absorción: (a) Agua - naranja de xilenol; (b) Znnaranja de xilenol

Dado que a esta longitud de onda se presenta una absorbancia significativa para la solución agua - naranja de xilenol y una relativa baja absorbancia para la solución Zn - naranja de xilenol. De esta manera trabajando a la longitud de onda seleccionada es posible detectar el punto final de la titulación espectrofotométrica.

Para la determinación de Zn en la muestra problema se pesan 0,5 g de Polvo Farm X, se disuelven con 1,50 mL de $\mathrm{HCl} 6 \mathrm{~mol} \mathrm{~L}^{-1}$ y se lleva a volumen en matraz aforado de $1000 \mathrm{~mL}$. De ésta solución se toman $2,00 \mathrm{~mL}$ y se introducen en la cubeta del espectrofotómetro, se agregan $0,50 \mathrm{~mL}$ de solución reguladora y $0,10 \mathrm{~mL}$ del indicador.
Se comienza a titular agregando desde la micropipeta solución de EDTA en cantidad equivalente al $70 \%$ de la cantidad requerida ${ }^{8}$. Se cubre la cubeta con un film, Parafilm M, y se agita manualmente por 10 segundos, se realiza la lectura espectrofotométrica de absorbancia a $414 \mathrm{~nm}$. Se continúa la titulación agregando cada vez volúmenes de $15 \mu \mathrm{L}$ y agitando después de cada adición hasta viraje del indicador y algunos $\mu \mathrm{L}$ en exceso para completar la curva de titulación. Se realizan varias determinaciones con distintos pesos de muestras del medicamento.

\section{RESULTADOS Y DISCUSIÓN}

En la Figura 2 se observa la curva de titulación para una de las determinaciones realizadas, correspondiente a un peso del polvo comercial Farm X de 0,4956g. Como se indicó anteriormente la constante de estabilidad del complejo Zn-EDTA es mayor que la constante de estabilidad del complejo $\mathrm{Zn}-\mathrm{XO}$, por lo tanto la primera especie es más estable.

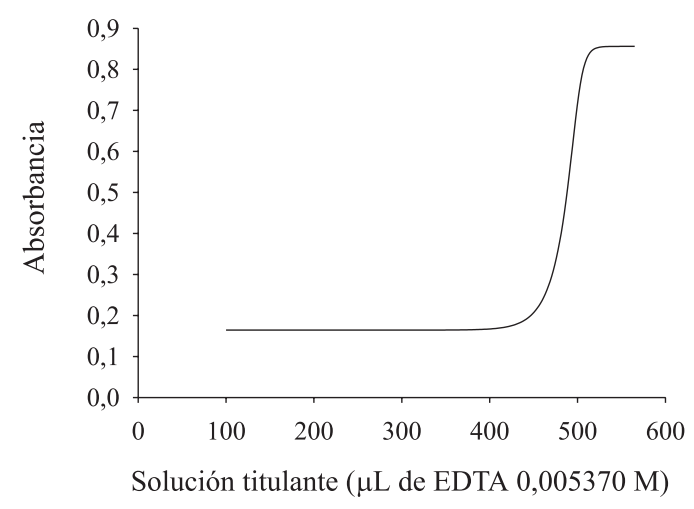

Figura 2. Curva de valoración de muestra de medicamento

A medida que la titulación progresa, el EDTA adicionado reacciona con el $\mathrm{Zn}$ (II) del complejo $\mathrm{Zn}-\mathrm{XO}$, formando el complejo Zn-EDTA y liberando el indicador. En el primer tramo de la curva se observa que las lecturas de absorbancia son prácticamente constantes debido a que mientras exista $\mathrm{Zn}$ (II) predomina el color del complejo $\mathrm{Zn}-\mathrm{XO}$. Al acercarse al punto de equivalencia donde todo el Zn se encuentra como complejo Zn-EDTA las lecturas de absorbancia comienzan a aumentar, produciendo un salto en su valor, debido al cambio de color del indicador liberado. Después del mencionado punto de equivalencia los valores de absorbancia permanecen aproximadamente constantes debido al predominio de color del indicador.

Cuando ya se cuenta con la curva de titulación, aparece un elemento subjetivo en el procedimiento. El analista debe determinar el punto de inflexión de la curva, con algún tipo de inspección. Puede trazar una línea vertical a partir del punto de inflexión de la curva hasta encontrar la intersección con el eje del volumen. Existe cierta inexactitud en este procedimiento que se reflejará en la lectura del volumen. Más exacto es obtener la representación gráfica de la pendiente de la curva de titulación, esto es el cambio de absorbancia con el cambio de volumen $(\Delta \mathrm{A} / \Delta \mathrm{V})$ contra el volumen del titulante, procedimiento denominado derivada primera. La curva que resulta tiene un máximo en el punto de equivalencia ${ }^{18}$.

En la Figura 3, se observa la representación gráfica para el caso analizado, de la cual se establece que el punto de equivalencia se alcanza a un volumen de EDTA de 497,5 $\mu \mathrm{L}=0,4975 \mathrm{~mL}$. Con este dato y siendo la concentración de EDTA $0,005370 \mathrm{~mol} \mathrm{~L}^{-1}(0,005370$ mmol $\mathrm{mL}^{-1}$ ), la masa molar del $\mathrm{Zn} 65,39 \mathrm{mg} / \mathrm{mmol}$ y el peso de la muestra problema 495,6 mg, recordando además que la reacción de 
valoración es 1:1, esto es mmol de EDTA $=$ mmol de $\mathrm{Zn}$, se obtiene: 0,4975 mL de EDTA x 0,005370 mmol/mL de EDTA x 65,39 mg de $\mathrm{Zn} / \mathrm{mmol}=0,1747 \mathrm{mg}$ de $\mathrm{Zn}$

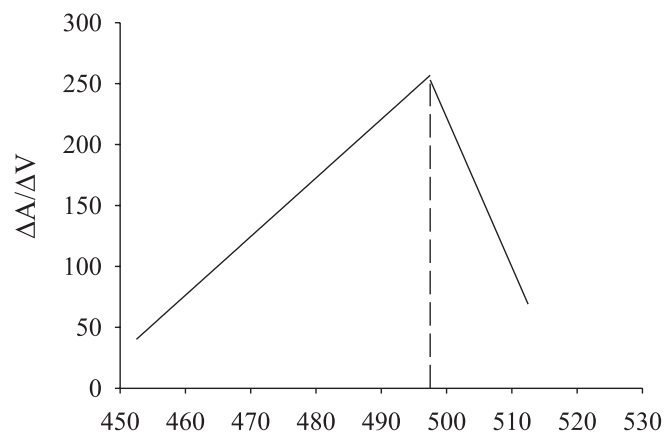

Solución titulante ( $\mu \mathrm{L}$ de EDTA $0,005370 \mathrm{M})$

Figura 3. Derivada primera de la curva de titulación

Esta cantidad esta presente en los 2,00 mL de solución analizada, de un total de $1000 \mathrm{~mL}$ que contiene los 495,6 mg de la muestra problema. Por lo tanto:

0,1747 mg de $\mathrm{Zn} / 2 \mathrm{~mL}$ de solución x $1000 \mathrm{~mL}$ de solución $/ \mathrm{L}=$ $87,35 \mathrm{mg}$ de $\mathrm{Zn} / \mathrm{L}$

$87,35 \mathrm{mg}$ de $\mathrm{Zn} / 495,6 \mathrm{mg}$ muestra $\mathrm{x} 100 \mathrm{mg}$ de muestra $=17,62 \%$ de $\mathrm{Zn}(\mathrm{m} / \mathrm{m})$

Otro camino para el cálculo es trabajar con el número de moles, entonces:

0,4975 mL de EDTA x 0,005370 mmoles/mL de EDTA x $1 \mathrm{~mol}$ de EDTA $/ 1000$ mmol de EDTA $=2,672 \times 10^{-6}$ moles de EDTA $=$ moles de $\mathrm{Zn}$

esta cantidad de moles está presente en $2,00 \mathrm{~mL}$ de muestra, pero se debe referir al volumen total de muestra, por lo tanto:

2,672 $\times 10^{-6}$ moles de $\mathrm{Zn} / 2,00 \mathrm{~mL}$ de solución $\mathrm{x} 1000 \mathrm{~mL}$ de solución $=1,336 \times 10^{-3}$ moles de $\mathrm{Zn}$

este es el total de moles de Zn que contiene la muestra pesada, se debe referir a $100 \mathrm{~g}$ de muestra para obtener el\% de $\mathrm{Zn}$, entonces: $1,336 \times 10^{-3}$ moles de $\mathrm{Zn} / 0,4956 \mathrm{~g}$ muestra $\times 100 \mathrm{~g}$ de muestra $=0,2695$ moles de $\mathrm{Zn}$

teniendo en cuenta la masa molar del $\mathrm{Zn}=65,39 \mathrm{~g}$ de $\mathrm{Zn} / \mathrm{mol}$ se tiene:

0,2695 mol de Znx65,39 g de $\mathrm{Zn} / \mathrm{mol}=17,62 \%$ de $\mathrm{Zn}(\mathrm{m} / \mathrm{m})$

En la Tabla 1 se observan los resultados obtenidos para el medicamento Polvo Farm X en una serie de cinco determinaciones.

La estadística descriptiva para estos datos, al nivel del $95 \%$ de confianza indica: media $\bar{X}=17,26$; desvío estándar s $=0,54$ y límite de confianza $\Delta X=0,67$ realizados con Excel 97.

Este valor, comparado con la información suministrada en la etiqueta del medicamento que indica el $20 \%$ de óxido de cinc y $10 \%$ de undecilenato de cinc, que se traduce en un $17,56 \%(\mathrm{~m} / \mathrm{m})$ de cinc,

Tabla 1. Porcentaje de cinc obtenidos para diferentes muestras

\begin{tabular}{ccc}
\hline $\begin{array}{c}\text { Peso de } \\
\text { Muestra }(\mathrm{g})\end{array}$ & $\begin{array}{c}\text { Volumen de E.D.T.A } \\
(\mu \mathrm{L})\end{array}$ & $\%$ de $\mathrm{Zn}$ \\
\hline 0,5021 & 480,0 & 16,78 \\
0,5062 & 500,0 & 17,33 \\
0,4917 & 502,5 & 17,93 \\
0,4956 & 497,5 & 17,62 \\
0,5024 & 477,0 & 16,66 \\
\hline
\end{tabular}

demuestra que la diferencia entre el valor experimental y el valor supuesto real no es significativa de acuerdo a la expresión estadística ${ }^{17}$ :

$t=\frac{\bar{X}-X_{m}}{s / \sqrt{N}}$

Donde t es el valor calculado, $\mathrm{X}$ es la media experimental, $\mathrm{X}_{\mathrm{m}}$ es valor supuesto real, s el desvío estándar y $\mathrm{N}$ el número de determinaciones. Este valor calculado indica 1,24 mientras que el valor de t de Student para el $95 \%$ de confianza es de 2,78, por lo tanto, dado que $|\mathrm{t}|<\mathrm{t}$ crítico, la diferencia no es significativa.

Se realizo la determinación de cinc, por medio de una técnica de espectrometría de emisión atómica por plasma inductivo, para la misma muestra arrojando un resultado de $18,10 \pm 0,20 \%$ de $\mathrm{Zn}$.

\section{CONCLUSIONES}

Los estudiantes debieron familiarizarse con el uso de instrumental moderno. El método propuesto es adecuado y estadísticamente aceptable, además resultó altamente motivante tanto para los alumnos como para los integrantes de la cátedra.

Instaló la discusión en el aula de temas tales como: titulaciones espectrofotométricas con indicadores y de mezclas de metales, reducción a microescala de los métodos convencionales de laboratorio, generación de menor cantidad de desechos y de residuos peligrosos.

Quizá el aporte adicional de este trabajo es la propuesta de la utilización de las técnicas a microescala, que cada día adquieren mayor importancia en la enseñanza de la química, por su economía, por su bajo impacto ambiental, por que permiten el desarrollo de habilidades en el cuidado y manejo de sustancias químicas y obtener de esta manera mayor seguridad. En síntesis presenta una forma de enseñar que respeta a las personas involucradas y a su entorno.

\section{REFERENCIAS}

1. http://www.who.int/pcs/ehc/summaries/ehc_221.html, introducida en Junio 2001.

2. Baran E. J.; Química Bioinorgánica, Mc Graw Hill Interamericana de España S.A: Madrid, 1995.

3. Manouri, O. Ch.; Papadimas, N. D.; Salta, S. E.; Ragos, G. Ch.; Demertzis, M. A.; Issopoulos, P. B.; Il Farmaco 1998, 53, 563.

4. Gaubeur, I.; da Cunha Areias, M. C.; Ávila Terra, L. H.; Suárez-Iha, M. E.; Spectrosc. Lett. 2002, 35, 455.

5. Salvador, A.; Pascual - Martí, M. C.; Adell, J. R.; Requeni, A.; March, J. G.; J. Pharm. Biomed. Anal. 2000, 22, 301.

6. Locatelli, C.; Torsi, G.; J. Electroanal. Chem. 2001, 509, 80.

7. Singh, M.; McGowan, C.; Szafran, Z.; Pike, M.; J. Chem. Educ. 2000, 77, 625 .

8. Gordon, J. S.; Harman, S.; Weis, K.; Pettegrew, B.; J. Chem. Educ. 2001, $78,1089$.

9. Richardson, J.; Stauffer, M.; Henry, J.; J. Chem. Educ. 2003, 80, 65

10. Torres Espinosa, E.; Castrellón Santa Ana, J. P.; Educ. Quím. 2000, 11, 262.

11. Merck; Reactivos orgánicos para el análisis inorgánico, Industria Gráfica S.A.: Barcelona, 1972.

12. Študlar, K.; Janoušek, I.; Talanta 1961, 8, 203.

13. van Staden, F. J.; Tlowana, I. S.; Talanta 2002, 58, 1115.

14. Benamor, M.; Belhamel, K.; Draa, T. M.; J. Pharm. Biomed. Anal. 2000, 23, 1033.

15. Kuroda, R.; Mochizuki, T.; Talanta 1981, 28, 389.

16. Pribil, R.; Analytical Application of EDTA and Related Compounds, $1^{\text {st }}$ ed., Pergamon Press: Germany, 1972.

17. Skoog, D. A.; West, D. M.; Holler, F. J.; Crouch, S. R.; Química Analítica, $7^{\mathrm{a}}$ ed., Mc Graw-Hill: México, 2001.

18. Day, Jr. R. A.; Underwood, A. L.; Química Analítica Cuantitativa, $5^{\text {a }}$ ed., Prentice - Hall Hispanoamericana, S.A.: México, 1991. 